\title{
The role of the IACUC in ensuring research reproducibility
}

\author{
Jerald Silverman ${ }^{1}$, James Macy² \& Patricia A Preisig ${ }^{3}$
}

There is a "village" of people impacting research reproducibility, such as funding panels, the IACUC and its support staff, institutional leaders, investigators, veterinarians, animal facilities, and professional journals. IACUCs can contribute to research reproducibility by ensuring that reviews of animal use requests, program self-assessments and post-approval monitoring programs are sufficiently thorough, the animal model is appropriate for testing the hypothesis, animal care and use is conducted in a manner that is compliant with external and institutional requirements, and extraneous variables are minimized. The persons comprising the village also must have a shared vision that guards against reproducibility problems while simultaneously avoids being viewed as a burden to research. This review analyzes and discusses aspects of the IACUC's "must do" and "can do" activities that impact the ability of a study to be reproduced. We believe that the IACUC, with support from and when working synergistically with other entities in the village, can contribute to minimizing unintended research variables and strengthen research reproducibility.

In recent years there has been increasing awareness and concern about the difficulty in obtaining reproducible data when the same experiment is repeated in a laboratory other than the laboratory where it was originally conducted. The extent of this problem is underscored by publications, surveys, and workshops devoted to the topic ${ }^{1-4}$. The problem extends beyond biomedical experimentation as similar concerns have been raised for research conducted in psychology $\mathrm{y}^{5,6}$, ecology $^{7}$, nutrition ${ }^{8}$, bioinformatics ${ }^{9}$, human clinical medicine ${ }^{10}$ and homeopathic disciplines ${ }^{11}$.

Improving reproducibility success takes the effort of an entire "village" that includes entities such as funding review panels, investigators, research institution leadership, laboratory animal facilities, IACUCs, and journals. Using an example emanating from an institution's animal program, the inability to reproduce experimental results may be due to nothing more than one unintentional experimental variable that might seem innocuous at first glance, such as temperature variations, exposure to different microbiomes, or surgical technique variations. Fortunately, safety nets already exist to help guard against such seemingly innocuous variables, and when properly employed they can individually and collectively enhance the likelihood of a study's reproducibility. For instance, a) an organization's leadership can set an example for, and insist upon, the highest level of research integrity, including institutional expectations for regulatory compliance and principled support for compliance committees, b) principal investigators can provide more detailed and coherent animal procedure protocols and provide adequate oversight of laboratory operations that can include assuring that reagents or diets are in date and properly stored, animals are correctly identified, study endpoints are clearly understood and applied, all needed records are retained, and staff members are appropriately and adequately trained, c) animal facilities can ensure a consistently high level of animal husbandry, veterinary care and recordkeeping practices, including records of the vendors used to purchase commodities as well as records of the food, bedding, room environmental conditions and other variables that have the potential to impact the reproducibility of a study, d) IACUCs can ensure that reviews of animal use requests, program self-assessments and post-approval monitoring programs are sufficiently thorough to reasonably ensure that the animal model is appropriate for testing the hypothesis and that animal use is conducted in a manner that is compliant with external and internal requirements. Thus, IACUC responsibilities must synergize with the rest of the institutional animal program to minimize unintended variables that can impact reproducibility.

Outside of an institution's animal program, a) federal study sections or other review panels should require a thorough description

${ }^{1}$ Department of Animal Medicine and Department of Pathology, University of Massachusetts Medical School, Worcester, Massachusetts. ${ }^{2}$ Yale Animal Resources Center

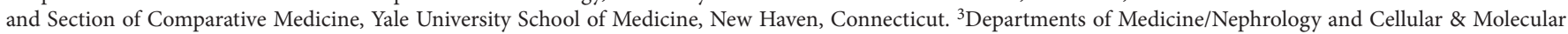
Physiology, Yale University, New Haven, Connecticut. Correspondence should be addressed to J.S. (Jerald.silverman@umassmed.edu). 
and evaluation of the chosen model, proposed study design, and specific procedures involved and b) professional journals should establish and enforce criteria for manuscript acceptance, including those suggested in the ARRIVE ${ }^{12}$ and CONSORT ${ }^{13}$ guidelines.

The difference between what the IACUC can do from what it must do should be recognized. Whether it is referred to as the "high road" versus the "low road", the spirit versus the "letter of the law" or, most likely, some combination thereof, each institution has a culture that influences its approach to regulatory oversight and research integrity. However, it is the IACUC that has the latitude to set performance standards and, therefore, set the stringency with which it performs its mandated duties. Clearly, as investigators have increasing pressures on their time, their concerns about regulatory burden and creep must be acknowledged and managed when establishing the level of checks-and-balances applied and safety nets implemented in the oversight of animal-based research. Thus, the IACUC's role in reproducibility can be broad, but ultimately boils down to the institution's philosophical approach to addressing regulations and research integrity. The remainder of this article will focus on what IACUCs can do to promote experimental reproducibility within the context of its mandated responsibilities.

However, as alluded to above, the IACUCs' efforts are only one of the safety nets needed to maximize the chance that an experimental finding can be reproduced. Indeed, the committee's efforts will be greatly diminished or even negated if the other entities supporting, reviewing, approving, disseminating and otherwise contributing to animal-based research do not ensure that their own safeguards are in place and free from ambiguity.

\section{The IACUC's role in the choice of an animal model}

One of the first decisions an investigator has to make when designing an experiment using animals is to select the appropriate model. The public has a right to expect that the model will advance knowledge and have societal value. If the proposed work has not undergone peer review, IACUCs are obligated to confirm that the model choice is supported by sound, objective and logical reasoning ${ }^{14}$. Animal models can be used to study normative biology or behavior or a pathological process having one or more attributes resembling the condition in humans or other animals. Realistically, most animal models have limitations and the validity and applicability of a model to predict responses in another species may or may not be consistent or reproducible. In other words, model reproducibility may arise independently or concurrently with model validity.

Model reproducibility can also be dependent on the natural biological variability of the animal population, which is an intrinsic characteristic of live animal models. In addition, model reproducibility can be impacted by extrinsic factors such as environmental variables, infections, medications and experimental technique. These and other factors should be considered by the IACUC and shortcomings in the description of animal use should be addressed before IACUC approval. Review of such factors is at the core of the IACUC's responsibilities related to animal use approval, and it dovetails with issues affecting the accuracy and reliability with which a study can be reproduced by another investigator. These extrinsic and intrinsic factors are a major focus of the remainder of this article.

\section{The importance of committee composition}

In a theme similar to the village analogy described earlier, a committee that is comprised of members with a broad array of expertise and perspectives is more likely to detect flaws in proposed animal use compared to a minimally constituted committee. Furthermore, the greatest potential for IACUC decision making to contribute to experimental reproducibility occurs when a robustly constituted committee's deliberations emanate from a discussion where the viewpoints of all participants are heard, i.e., the scientists, veterinarians, non-scientists, unaffiliated members and those with other expertise deemed necessary by the IACUC. These discussions are also helpful for all members by serving as a mechanism to establish and maintain an informed, experienced IACUC.

Although using the Designated Member Review (DMR) process, as established by the federal Office of Laboratory Welfare (OLAW), is within the letter of the regulations for protocol review, it often circumvents potentially valuable discussions. While it is true that use of DMR requires that all committee members have access to the research protocol, reviewing a document in isolation (either as the designated reviewer or as a committee member who has to determine if a Full Committee Review should occur) is unlikely to result in the same level of engagement with other IACUC members or expose the membership to the type and depth of discussions that could impact the potential for reproducibility issues. Thus, given the nuances that can impact reproducibility, it is important that the collective expertise and perspectives of the committee be fully leveraged for a beneficial review of the protocol. However, the benefits of such deliberations have to be balanced with the need to manage the workload of individual committee members, which is often accomplished by shortening convened meetings of the full committee and increasing the number of protocol or amendment reviews conducted primarily by IACUC office staff or a subcommittee of the IACUC and the IACUC office. A likely unintended outcome of these trends is that committee members will increasingly lack exposure to the type and depth of discussions that are needed for them to be strong contributors to protocol review and, perhaps more bothersome, the "village" will become less of an active participant in animal use approval-a mandated IACUC responsibility. As mentioned above, less experienced, less knowledgeable reviewers, or limited review perspectives, are less likely to identify factors that could adversely impact experimental reproducibility.

\section{IACUC oversight of approved activities}

Those individuals conducting program self-assessment activities such as facility inspections, program reviews and post-approval monitoring activities should not be working in silos. Rather, such teams should be composed of individuals with different expertise and perspectives, each of whom has a good sense of the regulatory requirements, institutional program standards and the aspects of individual experiments that are most critical for ensuring reproducibility. This last item includes proper and consistent surgical technique, record keeping, therapeutic drug use, study endpoints, euthanasia criteria, model creation, the appropriate animal genotype, environmental conditions, veterinary care, husbandry practices, and so forth. In addition, the same team of individuals should not always conduct the same activity and a given individual 
should not always perform an activity with the same team members. Having these activities performed by the IACUC's "village" leads to valuable diverse experiences, knowledge and skill sets.

As an example of the importance of IACUC oversight, consider post-approval monitoring. While the primary purpose of postapproval monitoring is to ensure that animal use is occurring as described in the approved protocol, failure to reproduce an experiment may be an unintended consequence of noncompliance. Thus, reviewing the space, procedures, and context in which animals are used helps guard against preventable reproducibility failures. Even though post-approval monitoring often has a negative connotation, given its link to regulatory compliance, it may identify potential issues affecting reproducibility that extend beyond compliance concerns and, in this context, it should be promoted as a positive enabling resource. In other words, post-approval monitoring presents an opportunity to remind investigators of the interdependencies between compliance, consistency and reproducibility.

In summary, the IACUC can clearly contribute more than just "checking off the box" for each of the federal requirements, but in doing so has to balance its stringency with increasing concerns of burden and the potential to promote tension between the entities involved in animal use, i.e., the IACUC, animal facility staff, veterinarians and investigators. Below are specific examples that fall under the IACUC's umbrella that are likely to impact study reproducibility.

The value of a harm:benefit analysis to advancing knowledge Although animal-based research supported by public or private agencies that rely on peer review to prioritize funding has, in theory, been evaluated for the scientific merit of the proposed studies, IACUCs have the responsibility to consider in its deliberations a harm:benefit analysis to help assess the study's contribution(s) to improved health of humans or other animals ${ }^{15}$. However, if the proposed work has not undergone peer review, IACUCs are obligated to conduct a more comprehensive review of the scientific soundness of the proposed work, including the proposed study design, use of appropriate animal models (discussed above), inclusion of both control and experimental groups, and the potential for experimental agents to address the study's hypothesis. Unless such scientific details and justifications are in the description of the proposed animal use, the IACUC cannot conduct an informative harm:benefit analysis. To the extent that such an analysis maximizes the chance for the experiment to be reproducible and, by extension, its potential to provide societal benefit, failure to conduct an informative harm:benefit analysis reduces the ability to assess societal benefit.

\section{Humane and study endpoints}

There are several issues included in the topics of humane and study endpoints that are relevant to all studies involving live vertebrate animals. These issues are independent of study design (experimental, observational), experimental setting (laboratory bench, field) or discipline (biological, environmental, ecological, societal). As related to experimental reproducibility, there are well known physiological changes that occur when animals are experiencing pain or distress, which are variables that can impact study results, especially when the definition and application of humane endpoints differ.
Another issue is the IACUC's approach to humane endpoints. Recently, there has been much discussion surrounding the use of surrogate markers of death to help define study endpoints ${ }^{16-20}$. While there are no published data on surrogate markers for every experimental model, blanket arguments against using surrogate markers or a lack of attempts to find a surrogate marker are unlikely to be acceptable. For example, if the research question is to determine if a given therapeutic treatment extends the life of the animal, a positive finding would typically be life extension on the scale of days, weeks or months, but not hours. Therefore, if animals are exhibiting clinical signs consistent with imminent death (on the scale of hours or perhaps a day), euthanizing those animals when such signs are exhibited will not likely have a significant impact on the conclusions of the study, yet it will end any pain, discomfort or distress that otherwise would be experienced by the animals as they die over the ensuing hours or day and perhaps result in more consistency in the final data. The key to successfully reproducing such a study is that the parameters assessed and used as clinical signs of impending death, and the associated euthanasia criteria, are as objective as possible, clearly described and consistently used. The IACUC can help ensure this through protocol review and post-approval monitoring.

There are also, of course, considerations of alternatives that can address the alleviation of pain, discomfort and distress-conditions well known to impact normal physiological systems and potentially influence the parameters under study. Failure to remove or minimize such factors increases the difficulty of creating a model that lends itself to being replicated by another investigator. This is the value of a stringent consideration of alternatives to refine experimental paradigms and result in the least number of potentially interfering factors before approval of the animal use request.

While a true alternative search is currently required only for studies involving USDA-covered species, the spirit of the alternative search is consistent with the requirement for addressing the 3 Rs for any study involving live vertebrate animals ${ }^{15,21}$. Because alternative search expertise is not often the forte of the required IACUC membership, and the value of the search is highly dependent on the quality of the search, IACUCs need to encourage the active participation of an individual(s) with such expertise, such as a librarian. Unfortunately, there is a wealth of data within the scientific community that is never published-for example, negative findings-and, if made available through centralized databases, could potentially inform the experimental designer and aid in refining studies.

Lastly are the actual euthanasia methods to be used. It is within the purview of the IACUC to ensure that these methods are appropriate, clearly described, and properly and consistently implemented. The potential impact of the euthanasia method is greatest when in vitro assays will be performed on harvested body parts, tissues or fluids. And, while the specific in vitro assays need not be described in the IACUC protocol, the rationale for using the animal to obtain biological samples for such assays must be justified; thus, the IACUC should address the potential impact of the euthanasia method to reduce the risk of exposing the biological samples to an extraneous variable(s). 
The current American Veterinary Medical Association euthanasia guidelines provide IACUCs with species-specific guidance for studies typically performed in a laboratory setting ${ }^{22}$. However, IACUCs often have less experience with euthanasia methods for species and settings not typical in most laboratories, such as wild animals in a field setting. For these situations, the IACUCs should seek guidance from the taxon-specific organizations, such as the American Society of Mammalogists, ornithological societies, American Fisheries Society, etc., as to the best approach to ensure the implementation of methods likely to be used by other investigators when replicating the study ${ }^{23}$.

\section{Experimental agents and therapeutic drugs}

There are two aspects to this topic that require IACUC attention. The first is the experimental protocol, including dose, route, frequency and duration of action of the experimental or therapeutic compound that the animal will receive, and how the animal will be monitored for adverse effects from the agent(s). The second aspect is to know the details of the agents or drugs and their appropriateness for their intended use, including how they may impact the study output data. Without such information provided in the protocol and the appropriate expertise of IACUC reviewers, the chances for successfully reproducing the experiment may be diminished.

There are some fairly easy checks and balances that the IACUC can use to maximize the chance that a study can be replicated when experimental agents and therapeutic drugs are involved. These include ensuring the sterility of parenterally administered agents or drugs, appropriate compatibility of administered biological materials, nutritional quality and storage of special diets, use of in-date agents or drugs, consistent drug reconstitution and storage methods, and the use of the appropriate grade of the administered agents or drugs. Proprietary experimental agents often present a challenge because there may be little information available and, in fact, the purpose of the study may be to obtain such information about the experimental agent (for example, to define the clinical signs of toxicity). Yet, such information is necessary to assist with reproducibility, which is essential because the ultimate goal of these pre-clinical studies may be future translational activities, such as first-in-human studies. Thus, before approving the use of experimental agents, IACUCs should request whatever information is available about the experimental agent and the procedure(s) to be used to prepare, administer and store the compound, and monitor the animal following administration of the compound. If providing the IACUC with the information that is known is complicated by the confidentiality of proprietary information, the institution can help the IACUC by either requiring such information in the contract project description, obtaining a commitment that such information will be disclosed to the IACUC before the study is approved, or at the least, an indication of the general class of the agent (for example, an ACE inhibiting drug) and what is known about the physiological impact of the agent compared to other members of the class. The importance of such information relates to replicating the study at a later time point when more information about the agent might be known.

\section{Animal identification, genotyping, phenotypes and adverse events}

There is no uniform agreement as to how much animal identification information must be included in a protocol. The species-level requirement was not much of an issue until genetically modified animals, first mostly mice and now fish, became the predominantly used live vertebrate animal. Individual animal identification is fundamental to ensuring the genotype (and phenotype) is ascribed to the appropriate animal. Furthermore, routine, stringent genotyping is required to ensure that genetic contamination has not occurred and to document that animals with the desired genotype are being studied.

A sobering reality is that mice with the same coat color are virtually indistinguishable and require unique individual identification and genotyping to ensure that the control and experimental groups are of the appropriate genotype. Mice of the wrong genotype can be shipped by vendors or colleagues, and individuals or groups of mice can become interchanged during manipulations in ways that are not visibly apparent, such as mice being placed back in the wrong cage, or cage cards being switched. These are devastating mistakes that can undermine reproducibility but can be minimized through the protocol review process, post-approval monitoring, setting program standards for animal genotyping, and animal and cage identification. The Guide for the Care and Use of Laboratory Animals addresses this issue ${ }^{15}$.

Similar to not providing details about the experimental animals or models, not disclosing abnormal phenotypes or adverse responses can impact replication of an experiment. How such information is made known to investigators trying to replicate a study is the responsibility of the publishing scientist, but at a minimum the IACUC should know about adverse events and unanticipated outcomes when it deliberates the continued use of the animal model. The committee also has to rely on the animal care staff, investigators, self-assessment and post-approval monitoring activities to be kept fully informed.

\section{Statistical and related considerations}

Requesting and using the appropriate number of animals, using animals of one or both sexes, and the random assignment of animals to study groups are significant considerations for research reproducibility and areas where the IACUC plays an important role. Reproducibility may suffer when too few animals are used in experimental groups, as it may be difficult to determine if a negative finding is real or the consequence of an underpowered experiment or small biological effect ${ }^{24}$. Conversely, although possibly not as influential on reproducibility, if more animals than are actually needed are used in a study, then more animals than needed will be exposed to the impact of the experimental paradigm, including pain or distress.

Appropriate number of animals in an experimental group In many experimental paradigms there are two issues that need to be addressed related to the justification for the total number of animals to be used. The first is the appropriate number of animals in each experimental group and the second is the number of times the experiment should be repeated on one or more groups. 
The former number should be statistically justified (see below), but the appropriate statistics for addressing the number of repetitions is not as clear. Nevertheless, the importance of a clear and defensible explanation for both of these issues is underscored by the information requested in the vertebrate animal section of Public Health Service grant applications and that the NIH Scientific Review Group has the authority to require that the number of animals being requested be further justified before an award is made ${ }^{25}$. Yet, despite a growing outcry for improved statistical design for animal-based research ${ }^{26-30}$ in a recent survey of nearly 1600 scientists from different life and physical science fields, nearly 55\% reported that low statistical power or poor statistical analysis always or often contributed to the inability to successfully reproduce an experiment, with another $35 \%$ reporting that these factors sometimes contribute to irreproducibility ${ }^{31}$.

Although IACUCs must evaluate the justifications for the number of animals being requested, not all scientists and IACUC members are sufficiently trained in biostatistics to know the appropriate statistical test to use, the needed sample size, or the most appropriate study design in order to determine the appropriate number of animals for each experimental group-all factors that can impact successful replication of the study. A recent survey found that only $31 \%$ of respondents reported that their IACUCs engage biostatistics specialists to help assure that the appropriate number of animals is being requested in protocol applications ${ }^{32}$. The presence of many internet sites that provide statistical help to researchers and IACUC reviewers seems to be of limited value, as most of these sites assume the user knows the appropriate test to use or already knows (or will be able to provide) certain statistical parameters, such as the standard deviation of a population sample. Due to such limitations, when a biostatistician or similarly trained person is available (and efforts should be directed toward having such an individual available) it behooves the IACUC to use that individual's services to review pertinent sections of protocols and act as an accessible resource for investigators and the IACUC.

Some IACUCs accept literature citations of the number of animals that were used in each experimental group in similar research, rather than requiring an inferential statistical justification specific for the study being proposed. Other IACUCs are more apt to follow the Guide for the Care and Use of Laboratory Animals, which states that "whenever possible, the number of animals and experimental group sizes should be statistically justified ..."15. Literature citations can provide useful guidance under certain circumstances, but experience suggests that such citations often are neither appropriate nor adequate to be used as the definitive source because either the statistical parameters applied or the experiments themselves are different, for example, studies having different endpoints. An IACUC policy on when and how literature citations can be used may prove helpful to some committees; however, such a policy should be more guidance than prescriptive to avoid the IACUC limiting investigator flexibility in addressing this issue. Thus, regardless of the approach, the rationale for the number of animals that make up an experimental group in a study must be such that an investigator trying to reproduce the study understands how the number was determined and is in agreement with that number.
Statistics-savvy protocol reviewers may wince when an investigator writes that additional animals will be added to an ongoing study in an attempt to reach statistical significance if the results do not reach statistical significance with the originally approved number of animals. Changing the group size can negatively impact study reproducibility unless the IACUC insists that appropriate statistical conditions are employed, such as a sequential stopping rule, and the investigator is transparent as to how the final group size was determined ${ }^{2}$.

\section{Number of times a study should be repeated}

IACUCs often review investigator requests to repeat their in vivo study in order to demonstrate its reproducibility in their hands. Such reproducibility is a hallmark of quality research, yet, as mentioned above, the appropriate statistical approach to determining that number is less clear and the IACUC may be asked to approve the number of repeats before the first cohort of animals has been studied. There is no simple answer as to how the IACUC should make a decision on these requests, but options include: a) suggesting that the investigator perform the study with a lesser statistical chance of obtaining a false positive result, such as by changing the significance level from the usual $P=0.05$ to $P=0.01$ to reduce the number of repeats needed should the results be positive; b) waiting until the study on the initial cohort of animals is completed before addressing the need for repetition; or c) allowing the requested number of repetitions to occur, based on the philosophy that no two experiments are ever conducted in exactly the same manner, even by the same investigators, so internal reproducibility has value because "significance [per se] is ... worthy of attention in the form of meriting more experimentation, but [is] not proof in itself" 33 . Most IACUCs would like to avoid having to address such considerations, but they must be addressed and, most importantly, decisions need to be made using a consistent set of criteria that are clear to the investigators. For a more in-depth discussion of the limitations to using statistical inferences for animal studies, the reader is directed to a recent book chapter on the subject ${ }^{34}$.

Investigator skill set and procedure performance consistency Although it is documented that many basic animal husbandry procedures can be stressful to laboratory animals ${ }^{35}$ and can impact phenotyping tests ${ }^{36}$, the technical skills of those performing research also can significantly influence the reproducibility of a study. However, mention of the skill level of an investigator or animal well-being difficulties resulting from a research procedure are unlikely to be found in most research presentations and publications, limiting the transparency as to how the study was actually conducted and whether there were adverse consequences attributable to investigator technique or skill.

Training is essential for personnel to develop and maintain the core technical competency necessary for maximizing performance consistency and, hence, reproducibility. Training of personnel in technical procedures, including the use of aseptic technique, anesthesia and analgesia (when appropriate), and tissue manipulation is an institutional responsibility that is shared by the principal investigator's laboratory, the vivarium staff and the IACUC. The IACUC's mandated role is to provide oversight and evaluation of 
the effectiveness of the institutional training program with the goal that animal use activities will lead to high quality scientific results and animal well-being ${ }^{15}$. Training is usually provided through instructional lectures, videos, and a wide spectrum of hands-on experiences. To fulfill its responsibilities, the IACUC needs to establish criteria for competency and should evaluate individual skill sets for having met those criteria for each procedure an individual will perform. This evaluation and ongoing monitoring can involve feedback from trainers and investigators, reports from the veterinary and laboratory staff, periodic reviews of training programs, laboratory and medical records, direct observation of ongoing procedures, and post-approval monitoring. Failure to evaluate or monitor technical competency leaves the IACUC without a method of determining the effectiveness of the institutional training program - one of an IACUC's core responsibilities.

Deficiencies such as a lack of adequate postoperative pain control, poor tissue handling during surgery, excessive intraoperative blood loss and inadequate aseptic technique are examples of stressors that can limit the ability of a study to be accurately replicated by another investigator-yet many such stressors are potentially preventable if an IACUC establishes, as indicated above, adequate criteria for evaluating an individual's technical ability, and the details of the technique used are correctly, clearly and thoroughly described in the protocol and in publications and presentations, and were followed as approved by the IACUC. A committee that simply accepts no more than a written statement that a person has performed a surgical procedure in the past and has retained adequate skills to continue performing it on the chosen species cannot be assured that the operator has even the minimal technical skills needed for the preparation of surgical instruments, use of aseptic technique, knowledge of suture materials and their use, tissue handling, surgical approaches, monitoring parameters and so forth. Often, the justification for the validity of such a statement is that there have not been any "adverse events" associated with the individual's work. While this may be so, adverse events are not the only indicators that experimental reproducibility may be limited. Therefore, it is incumbent upon an IACUC to develop, implement and periodically review training materials and procedures. The post-procedural monitoring programs used should also be periodically reviewed and updated as needed. Engagement in such activities can provide minimal assurance to the IACUC and other investigators that experimental procedures on live animal research subjects are not complicated by technical inaptitude.

\section{Housing and other environmental factors}

The ability of an IACUC to positively impact research reproducibility goes beyond model and animal selection, animal identification, statistical considerations, consistent procedures, and the other topics discussed earlier, although the potential impact of environmental factors may be less than for the prior topics. For example, the health status of the animal can lead to reproducibility issues and studies proposed in animal colonies with infections and concurrent health problems should be carefully evaluated for the known, suspected or likely impact of the pathogens or conditions on study results ${ }^{37-39}$. Other factors, such as cage overcrowding, may or may not be considered a stressor, depending on the study cited and an IACUC's own definition of overcrowding. Additional environmental stressors well-known to impact research outcomes include housing male mice or female hamsters together, which often leads to fighting and sometimes severe wounds. The macroand microenvironment, including temperature, humidity, noise, vibrations, lighting levels, environmental enrichment, water and food and bedding also are factors that can impact animals physiologically or behaviorally. Even subtle factors, such as the location of cages on the rack and the cage manufacturer may have an impact on the animals ${ }^{40}$.

In addition, recent attention has focused on the role of an animal's microbiome on its physiological state and responses to experimental maneuvers ${ }^{41}$. While relevant data may not be readily available to the IACUC, this is an area where veterinary or other expert input could be valuable. Such biologic variability can present as a reproducibility issue, especially given that it can synergize with, be masked by, or be interdependent of other factors affecting reproducibility. Thus, although these latter factors are not a primary focus of the IACUC, the vivarium should maintain records and make available to the IACUC and investigator the specific conditions of the animals' health and environment so, if needed, they can be accurately described in presentations or published data.

\section{Conclusion}

The issue of reproducibility transcends virtually all levels of the scientific enterprise. Using biological system models, in particular live, sentient animal models, introduces complex variables that complicate the potential for successful reproducibility of a study ${ }^{4,42}$. Selecting the most appropriate model, ensuring consistent experimental protocols and procedures, involving adequately trained and skilled investigators, and controlling extraneous variables will help minimize the risk of not being able to successfully reproduce an experimental finding - but this requires the diligence and cooperation of the entire scientific community. Clearly, the IACUC is in a position to bring factors impacting animal care and use issues to light and guard against them through thorough protocol reviews and program oversight, particularly when the "village" of people and perspectives that comprise the IACUC and its supporting staff are all involved.

These activities place the IACUC in a delicate position of straddling an amorphous intersection of animal welfare, scientific merit and scientific integrity, all of which can impact reproducibility. The IACUC's involvement in this breath of issues can be viewed as over-reach, creep, and burdensome to the investigator, making it very difficult to strike a balance between the strict interpretation of its prescribed regulatory mandates (the "must dos") and the spirit of IACUC involvement on behalf of knowledge advancement (the "can dos") from its unique position of being solely responsible for animal use approval and program oversight. The balance that is struck by the IACUC between the spirit of ensuring animal welfare and knowledge advancement and being viewed as a burden to other entities is often predicated on whether or not the institutional stakeholders have a shared vision for the approach to guarding against reproducibility problems. The absence of a shared vision can be a 
source of tension between the IACUC, investigators, laboratory animal facility, veterinarians and/or the institutional leadership. Ultimately, failure to address manageable factors that can erode reproducibility can undermine the value of animal-based studies and, similar to issues of perceived abusive animal care and use, poor experimental reproducibility can negatively affect public opinion about the value of animal-based research and the use of animals as research subjects.

\section{COMPETING FINANCIAL INTERESTS}

The authors declare no competing financial interests.

\section{Received 26 October 2016; accepted 23 December 2016}

Published online at http://www.nature.com/laban

1. Prinz, F., Schlange, T. \& Asadullah, K. Believe it or not: How much can we rely on published data on potential drug targets. Nat. Rev. Drug Discov. 10, 712 (2011).

2. Begley, C.G. \& Ellis, L.M. Drug development: Raise standards for preclinical cancer research. Nature 483, 531-533 (2012).

3. Dass, K.J., Scott, M.A., Galoforo, S.S., Loeffler, D.A. \& Coffey, M.P. Development of antihuman IgG antibodies and hematologic deficits but not clinical abnormalities in $\mathrm{C} 57 \mathrm{BL} / \mathrm{i} 6$ mice after repeated administration of human intravenous immunoglobulin. Comp. Med. 64, 169-170 (2014).

4. National Academies of Sciences, Engineering, and Medicine. Reproducibility Issues in Research with Animals and Animal Models: Workshop in Brief, Washington, DC: The National Academies Press, (2015) https://doi. org/10.17226/21835.

5. Vergés, A. et al. Personality disorders and the persistence of substance use disorders: A reanalysis of published NESARC findings. J. Abnorm. Psychol. 123, 809-820 (2014).

6. Open Science Collaboration. Estimating the reproducibility of psychological science. Science 349, 943-949. (2015).

7. Haddaway, N.R. \& Verhoeven, J.T. Poor methodological detail precludes experimental repeatability and hampers synthesis in ecology. Ecol. Evol. 5 (19), 4451-4454 (2015).

8. Sorkin, B.C., Kuszak, A.J., Williamson, J.S., Hopp, D.C. \& Betz, J.M. The challenge of reproducibility and accuracy in nutrition research: resources and pitfalls. Adv. Nutr. 7, 383-389 (2016).

9. Stegmayer, G., Pividori, M. \& Milone, D.H. A very simple and fast way to access and validate algorithms in reproducible research. Brief. Bioinform. 17, 180-183 (2016).

10. Snider, K.T., Johnson, J.C., Degenhardt, B.F., Snider, E.J. \& Burton, D.C. Association of low back pain, somatic dysfunction, and lumbar bone mineral density: reproducibility of findings. J. Am. Osteopath. Assoc. 114, 356-367 (2014).

11. Weingärtner, 0. A formal approach to the problem of reproducing experimental effects with homeopathic potencies. J. Altern. Complement. Med. 15, 287-291 (2009).

12. Kilkenny, C., Browne, W.J., Cuthill, I.C., Emerson, M. \& Altman, D.G. Improving bioscience research reporting: The ARRIVE guidelines for reporting animal research. PLOS Biol. 8, e1000412 (2010).

13. Schulz, K.F., Altman, D.G., Moher, D. \& CONSORT Group CONSORT 2010 Statement: Updated guidelines for reporting parallel group randomised trials. PLoS Med. 7, e1000251 (2010).

14. Office of Laboratory Animal Welfare. PHS Policy on Humane Care and Use of Laboratory Animals-Frequently Asked Questions. Protocol Review, Question No. D.12. Is the IACUC responsible for judging the scientific merit of proposals? (Washington, DC, 2006, revised 2016).

15. Institute for Laboratory Animal Research. Guide for the Care and Use of Laboratory Animals $8^{\text {th }}$ edn. (National Academies Press, Washington, DC, 2011).

16. Warn, P.A. et al. Infrared body temperature measurement of mice as an early predictor of death in experimental fungal infections. Lab. Anim. 37, 126-131 (2003).
17. Ray, M.A., Johnston, N.A., Verhulst, S., Trammell, R.A. \& Toth, L.A. Identification of markers for imminent death in mice used in longevity and aging research. J. Am. Assoc. Lab. Anim. Sci. 49, 282-288 (2010).

18. Solomon, J.A., Tarnopolsky, M.A. \& Hamadeh, M.J. One universal common endpoint in mouse models of amyotrophic lateral sclerosis. PLOS ONE 6, e20582 (2011).

19. Hankenson, F.C. et al. Weight loss and reduced body temperature determine humane endpoints in a mouse model of ocular herpesvirus infection. J. Am. Assoc. Lab. Anim. Sci. 52, 277-285 (2013).

20. Nunamaker, E.A., Artwohl, J.E., Anderson, R.J. \& Fortman, J.D. Endpoint refinement for total body irradiation of $\mathrm{C} 57 \mathrm{BL} / 6$ mice. Comp. Med. 63 , 22-28 (2013).

21. Russell, W.M.S. \& Burch, R.L. The Principles of Humane Experimental Research (Methuen, London, UK, 1959).

22. American Veterinary Medical Association. AVMA Guidelines for the Euthanasia of Animals. 2013 edition. https://www.avma.org/KB/Policies/ Documents/euthanasia.pdf (American Veterinary Medical Association, Schaumburg, IL, 2013).

23. Sikes, R.S. \& Gannon, W.L. \& the Animal Care and Use Committee of the American Society of Mammalogists. Guidelines of the American Society of Mammalogists for the use of wild mammals in research. J. Mammal. 92, 235-253 (2011).

24. Button, K.S. et al. Power failure: Why small sample size undermines the reliability of neuroscience. Nat. Rev. Neurosci. 14, 365-376 (2013).

25. National Institutes of Health. Clarification on the Roles of NIH Scientific Review Groups (SRG) and Institutional Animal Care and Use Committees (IACUC) in Review of Vertebrate Animal Research. Notice NOT-0D-10-128 (National Institutes of Health, Washington, D.C., September 2010).

26. Festing, M.F.W. Design and statistical methods in studies using animal models of development. ILAR J. 47, 5-14 (2006).

27. Scott, S. et al. Design, power, and interpretation of studies in the standard murine model of ALS. Amyotroph. Lateral Scler. 9 , 4-15 (2008).

28. Kilkenny, C. et al. Survey of the quality of experimental design, statistical analysis and reporting of research using animals. PLOS ONE 4, e7824 (2009).

29. Fitts, D. Ethics and animal numbers: Informal analyses, uncertain sample sizes, inefficient replications, and type I errors. J. Am. Assoc. Lab. Anim. Sci. 50, 445-453 (2011).

30. Hess, K.R. Statistical design considerations in animal studies published recently in Cancer Research. Cancer Res. 71, 625 (2011).

31. Baker, M. Is there a reproducibility crisis? Nature 533, 452-454 (2016).

32. Silverman, J., Baker, S.P. \& Litz, C.W. A self-assessment survey of the Institutional Animal Care and Use Committee. Part 1: animal welfare and protocol compliance. Lab Anim. (NY) 41, 230-235 (2012).

33. Goodman, S. A dirty dozen: Twelve p-value misconceptions. Semin. Hematol. 45, 135-140 (2008).

34. Gracely, E. Justification of the number of animals to be used. in The IACUC Handbook $3^{\text {rd }}$ edn. (ed. Silverman, J., Suckow, M. \& Murthy, S.) (CRC Press, Boca Raton, 2014).

35. Balcombe, J.P., Barnard, N.D. \& Sandusky, C. Laboratory routines cause animal stress. Contemp. Top. Lab. Anim. Sci. 43, 42-51 (2004).

36. Gerdin, A.K. et al. Experimental and husbandry procedures as potential modifiers of the results of phenotyping tests. Physiol. Behav. 106, 602-611 (2012).

37. Chichlowski, M. \& Hale, L. Effects of Helicobacter infection on research: the case for eradication of Helicobacter from rodent research colonies. Comp. Med. 59, 10-17 (2009).

38. Karst, M. \& Wobus, C. Viruses in rodent colonies: lessons learned from murine noroviruses. Annu. Rev. Virol 2, 525-548 (2015).

39. Baker, D.G. \& Lipman, N.S. Factors that can influence animal research. In Laboratory Animal Medicine, $3^{\text {rd }}$ edn (ed. Fox, J.G., Anderson, L.C., Otton, G., Pritchett-Corning, K.R., \& Whary, M.T.) (Academic Press, 2016).

40. Nevalainen, T. Animal husbandry and experimental design. ILAR J. 55, 392-398 (2014).

41. Bleich, A. \& Fox, J.G. The mammalian microbiome and its importance in laboratory animal research. ILAR J. 56, 153-158 (2015).

42. American College of Laboratory Animal Medicine. ACLAM position statement on reproducibility. J. Am. Assoc. Lab. Anim. Sci. 55, 824-825 (2016). 